\title{
Use of -la in Simple Sentences among Indian Ethnic group in Malaysia
}

\author{
Dr. Franklin Thambi Jose. S \\ Senior Lecturer, Faculty of Languages and Communication, Sultan Idris Education University, Malaysia \\ thambijoshs@gmail.com
}

\section{Doi:10.5901/mjss.2015.v6n6s2p122}

\begin{abstract}
Language is the ability of expressing ideas or thoughts of one's own. It varies according to the social structure of a local speech community. Moreover it expresses a group identity. The group can be a community, ethnicity, class or caste. A group of people who live in Malaysia speak Tamil and they are called as Indian ethnic group. This group includes Hindi, Telugu and Malayalam speakers. They form 7.1\% (National Census, 2000) of the total population. In Indian ethnic group, Tamil forms the largest subgroup (5.7\%). Although other language speakers are included in Indian ethnic group, it represents Tamil speakers. This group (subgroup) use -la when they speak Tamil language. Its literary meaning is 'dear' in English. According to Baron (1986) the minority language in a larger social group differs in pronunciation, usage, etc. Since Tamil group is living with Malay language speaking people, the usage of -la came to exit and is unavoidable. -la is used in simple sentences in different contexts. The different contexts are identified such as usage of simple sentences between friends, students, husband and wife, parents and children and immigrants. For example: vaa-la naaam poovoom. 'come dear, we shall go' (used between friends) The major objective of this paper is to analyse the usage of -la linguistically in simple sentences.
\end{abstract}

Keywords: Language, speech community, Tamil, Indian, ethnic group

\section{Introduction}

Language is the ability of expressing ideas or thoughts of one's own. It can be also said as, the way of communicating with each other. Language can be defined as the reflection of a society. It varies according to the social structure of a speech community. Moreover, it expresses a group identity. The group can be community, ethnicity, class or caste.

A group of people who live in Malaysia speak Tamil and they are called as Indian ethnic group. Indian ethnic group includes speakers of Malayalam, Telugu, Punjabi, Hindi and other languages speakers of India. They were migrants of India two generations back. They came to Malaysia as traders and estate labours. They are along with the other two ethnic groups such as Malay and Chinese. They form 7.1\% of the total population in Malaysia (National Census 2010). But Tamil language and culture was spread during the reign of Chola Dynasty around $11^{\text {th }}$ century AD.

\section{Tamil Language}

Tamil is one of the major Dravidian languages, which has a very long written history of more than 2000 years. It is mainly spoken in Tamilnadu (in India), Sri Lanka, Malaysia and Singapore. Apart from these, countries like Indonesia, IndoChina, Fuji, South Africa and British Guinea have many Tamil speaking communities. It is also spoken in islands like Mauritius and Madagascar. About 77 million Tamil people are living around the world. It is an official language in Tamilnadu (in India), Sri Lanka, Malaysia and Singapore. Tamil is one of the two classical languages in India viz Sanskrit and Tamil.

Tolkappiam is the oldest text among all the grammars in Tamil, which belongs to $200 \mathrm{BC}$ or around that period. This grammar is also considered to be one of the pioneer grammars known to the human history. Another significant and unique characteristic feature of Tamil is its diaglossic nature and also the prevalent maintenance of this distinguishion by the Tamil speaking community across the world to a great extent.

Tamil has its own scripts with 12 vowels, 18 consonants and 1 aaytam. 'Tamil script is descended from the southern Indian Pallava script' (Britannica Ready Reference, 2004; 203).

\section{Malaysia Tamil Speakers}

The Tamil speakers of Malaysia use Tamil language with a mixture of Malay and English languages in informal speeches. 
According to Baron (1986) the minority language in a larger social group differs in pronunciation, usage, etc. As mentioned earlier the Tamil speakers live along with Malay and Chinese language speakers. Of these Malay is the majority speaking language. The Tamil speakers learn Malay from their childhood i.e.standard-1 in schools. Though they speak good Malay, they miss the tone of the language as the native speaker does. Since Malay speaking situation is found in all places and it is the national language, the Tamils in Indian ethnic group tend to speak Malay mixed words in everyday Tamil. Moreover they adopt the usage of $-l a$ 'dear' in their daily usages in majority of the simple sentences they utter.

\section{Simple Sentences}

A simple sentence consists of a single clause with a subject and a predicate in general. But there are situations in which a subject or a predicate can also be a simple sentence. For example in English:

In a conversation, 'Oliver' or 'Came' can be a subject and predicate respectively.

In Tamil also the same can be used. The structure of the simple sentence in Tamil language is:

Subject (S) + Object (O) + Verb (V)

Though it is the standard simple sentence pattern, words can, to some extent, move freely in a sentence and hence it is said to be a free word ordered language. But all the words cannot be moved and it can be moved only within a phrase. So it can be called as free phrase ordered language (Thambi Jose, 2015). The following structures are possible in Tamil language:
Subject $(\mathrm{S})+\operatorname{Verb}(\mathrm{V})+$ Object $(\mathrm{O})$
$\operatorname{Object}(\mathrm{O})+\operatorname{Verb}(\mathrm{V})+$ Subject $(\mathrm{S})$
Object (O) + Subject (S) + Verb (V)
Verb $(V)+$ Subject $(\mathrm{S})+$ Object $(\mathrm{O})$
Verb (V) + Object (O) + Subject (S)
Consider the following examples in Tamil:
1. naan paadam paditeen (SOV) 'I studied the lesson.'
2. naan paditeen paadam (SVO) 'I studied the lesson.'
3. paadam paditeen naan (OVS) 'I studied the lesson.'
4. paadam naan paditeen (OSV) 'I studied the lesson.'
5. paditeen naan paadam (SVO) 'I studied the lesson.'
6. paditeen paadam naan (VOS) 'I studied the lesson.'

\section{Use of -la 'Dear' in Simple Sentences}

In daily activities, many simple sentences are used when compared to other type of sentences in almost all the languages. It is a common phenomenon that Malaysians when they speak Malay use -la 'dear' atleast in 70 simple sentences out of 100 other sentences they use. The following chart shows this:

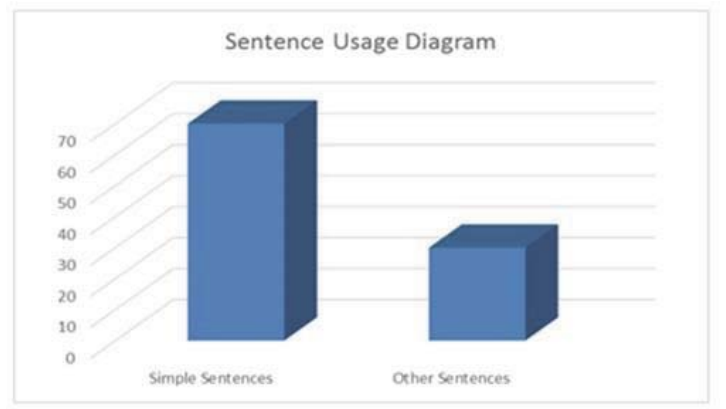

The Tamil speakers follow Malay speakers and use -la 'dear' in both English and Tamil. They use this in three different meanings in simple sentences.

\section{$>$ Emphasis}


-la 'dear' is used in simple sentences to emphasis certain things.

For instance, cikkanaa-la? 'Is it chicken, dear?'

$>$ Love and affection

To show one's closeness i.e. love and affection, he/she uses -la 'dear' in simple sentences.

For instance, naam pooma-la 'Shall we go, dear?'

$>$ Quick reaction

To express a quick reaction about something heard, -la 'dear' is used in simple sentences.

For instance, enna-la? 'what, dear?'

Due to these kinds of meanings in the usage of -la 'dear' in simple sentences, the study of the context plays an important role. To know the context in which it is used, it is significant to know about the occurrences. It is occurred inbetween friends, students, husband \& wife and parents \& in-between children.

\subsection{Friends}

When friends talk each other, they use -la 'dear' to show their affection and sometimes emphasis too. Most of friends replied they use -la 'dear' as a fashion, when the researcher asked them. The use $-l a$ 'dear' is given below in simple sentences:

1. enakku onnum puriyale-la 'Dear, I didn't understand anything.'

2. naaLaikku viiTTukku poReen-la 'Dear, tomorrow I am leaving to home.'

3. vaa-la koyilukku pooovoom 'Come dear, we can go to temple.'

4. -la konjam utavi pannu 'Help me dear.'

5. enakku tuukkam varutu-la 'I am feeling sleepy, dear.'

\subsection{Students}

In Malaysian context, Tamil students start studying Malay and Tamil languages from their childhood. Most of the Tamil speaking students have close relationship with Malay students. They speak to the Malay students in Malay language. In Malay schools the medium of instruction is Malay. So the conversation in classroom between the teacher and students will be in Malay language. This situation prevails from schools to colleges/universities. The Tamil students when they talk among themselves they use Tamil. In their Tamil, they use -la 'dear' in simple sentences. In colleges/universities the Tamil students studying in English departments use English and in their English they use -la 'dear'. Consider the following examples:

In Tamil:
1. nii padicciyaa-la?
'Did you study, dear?'
2. peena kodu-la 'Give your pen, dear'
3. tiiccar enna-la connaanga? 'What did the teacher tell, dear?'
In English:
1. Please-la help me. 'Please dear, help me.'
2. Wait-la, I am also coming. 'Wait dear, I am also coming.'
3. thank you-la 'Thank you dear.'

\subsection{Husband \& Wife}

In Tamil culture, wife should give more respect to her husband than anyone else. Even today in some Tamil families the wife never uses her husband's name to tell others. To build good family relationship between husband and wife they show their love and affection by using -la 'dear' in their daily conversations. Some examples are given below:
1. caappitiyaa-la?
'Did you eat, dear.'
2. raani ethavathu pesu-la 'Rani, talk something, dear.'
3. inka paru-la 'See here, dear.'

Here the use of $-l a$ 'dear' is clearly seen that to show the affection between husband and wife. But they use this even for emphasis. Consider the following examples:

1. kaalaiyile anju manikku ezuppu-la 'Call me at 5'o clock (dear).'

2. tii kodu-la

'Give me tea (dear).' 


\subsection{Parents \& In-between Children}

Family relationship can be divided into two parts. One is relationship between parents and children and another one between children, i.e. elder sister and younger sister, brother and sister and so on. Here also the usage of -la 'dear' prevails because of love and affection.

In between children the usage of $-l a$ 'dear' is more when compared with parents and children relationship. For instance;

In-between children:

1. akkaa inka vaa-la 'sister come here, dear.'

2. maalaa caappittiiyaa-la 'Mala did you eat, dear.'

3. enekku teriyale-la 'I don't know, dear.'

In-between parents and children:

1. kadaikku poyirru vaa-la 'Go to market dear.'

2. nallaa padikkanum-la 'You should study well, dear.'

3. een paecaama irukkira-la 'Why are not talking, dear.'

\section{6. -la 'dear' with Grammatical Categories}

-la 'dear' occurs with all major grammatical categories such as noun, verb, adjective and adverb. Consider the following examples.

With noun

pazama-la, kondu vaa 'Is it banana, dear'

With verb

vakuppukku poyirru vaa-la 'Go to class, dear.'

With adjective

enna azalaku-la 'It is beautiful, dear'

With adverb

ciikkiram-la 'fast, dear'

From the above examples one can surely tell that -la 'dear' occurs with all the major grammatical categories in Tamil language.

\section{Conclusion}

The use of -la 'dear' can be seen in simple sentences. That doesn't mean, it is found only in simple sentences, may be in other type of sentences too. Basically Tamil speaking people in Malaysia are multilinguist. They speak Malay, English, Tamil and a few can understand Chinese. Since they start learning Malay from early childhood from schools and neighbours, the use of $-l a$ 'dear' start from their childhood. By using -la 'dear' they assume that they maintain their relationships. But sometime it fails. For instance, a student who has good relation with his/her teacher cannot use -la 'dear'. In this context it cannot be used because of respect towards the teacher. The use of -la 'dear' is increasing day by day because of the prestige in the society. This suffix falls under the grammatical category called clitics, which is the part of particle. By using this, the peculiar sounds such as La \& za of Tamil are getting lost. Another notable is the usage of this is more in simple sentences.

\section{References}

Arasarattnam, S. (1970) Indian in Malaysia and Singapore, Kuala Lumpur, Oxford University press.

David, M.K. and Naji. (2001) Do Minorities have to Abandon their Language IL: A case study of Malaysian Tamils. International Scope review, 2 pg.1 -15), Malaysia.

Palmer, Frank. (1972) Grammar. London: Penguin Books.

Paramasivam, M and Mohan Lal, Sam. (2010) Patterns of Code Switching Among the Tamil Students of Malaysia. (Unpublished article) Sapir, Edward (1921) Language NewYork: Brace and World.

Subrahmanian, N (1996). The Tamils: Their History, Culture, and Civilization. Madras: Institute of Asian studies.

Thambi Jose, Franklin (2015) Issues in Developing Tamil Software for Malaysian Secondary Schools. Paper presented in International Conference held at UPSI. (Unpublished)

National Census (2010) Department of Statistics, Malaysia. 